\title{
Reversibility of histological and immunohistological abnormalities in sublabial salivary gland biopsy specimens following treatment with corticosteroids in Sjögren's syndrome
}

\author{
M M Zandbelt, F H J van den Hoogen, P C de Wilde, P J S van den Berg, \\ H G F Schneider, L B A van de Putte
}

\begin{abstract}
Sjögren's syndrome (SS) is a chronic autoimmune disease characterised by specific lesions in exocrine glands, so sublabial minor salivary gland biopsy (SLGB) plays an important part in its diagnosis. The extent and composition of the lymphocytic infiltrate in SLGB specimens can be considered as target organ specific parameters. They are quantified after histological and immunohistological examination by a focus score (describing the extent of the infiltrate) and IgA\% score (describing the composition of the infiltrate), respectively. However, little is known about the factors that contribute to the extent and composition of the infiltrate and whether these features are reversible as repeated SLGBs are rarely performed. A patient with SS is described who underwent SLGBs before and after treatment with high dose corticosteroids. After treatment there was not only clinical improvement, but also improvement in the histological and immunohistological parameters. Although these findings need to be confirmed in further studies, this suggests that histopathological changes may be reversible in SS. Furthermore, it shows that the potential effects of corticosteroid use should be taken into account when interpreting SLGB specimens. When clinical changes do parallel histological changes, repeated SLGBs might offer a marker for disease activity in patients with SS.

(Ann Rheum Dis 2001;60:511-513)
\end{abstract}

epartment of

Ophthalmology,

Elkerliek Hospital,

Helmond, Netherlands

H G F Schneider

Correspondence to:

Dr M Zandbelt, Department

of Rheumatology, UMC St

Radboud, Nijmegen, P O

Box 9101, $6500 \mathrm{HB}$

Nijmegen, Netherlands

M.Zandbelt@reuma.azn.nl

Accepted 30 November 2000 patients with primary SS - that is, without comorbidity from other rheumatic diseaseshave an increased risk of developing a nonHodgkin's lymphoma which is 44 times greater than the rate in the general population. ${ }^{2}$ Since the first description of SS by Henrik Sjögren, many criteria have been proposed for the diagnosis or classification of this syndrome..$^{3-6}$ Histological examination of sublabial minor salivary gland biopsy (SLGB) specimens is an important factor in these criteria. The extent and composition of the lymphocytic infiltrate in the SLGB specimens can be considered as target organ specific parameters. The number of lymphocytic foci per $4 \mathrm{~mm}^{2}$ in the SLGB specimen is expressed as a focus score. However, it is well known that the focus score lacks both sufficient sensitivity and specificity for the diagnosis of SS, depending on whether a focus score of $\geqslant 1$ (leading to decreased specificity) or $\geqslant 2$ (leading to decreased sensitivity) is taken as the criterion for diagnosis. ${ }^{7-9}$ In contrast to the focus score, immunohistological examination of the infiltrate has been shown to have both high disease specificity and sensitivity. ${ }^{710}$ This method is based on quantification of plasma cells expressing different isotypes of immunoglobulins (IgA, IgM, IgG). A percentage of IgA containing plasma cells (\% IgA) of less than 70, or of IgM containing plasma cells (\% IgM) of more than 10 , is highly specific for SS. So far, little is known about the factors that contribute to the lymphocytic infiltrate. Manthorpe et al recently reported the possibility that patients might "smoke" their lymphocytic infiltrate away. ${ }^{11}$ In their study the focus scores found in SLGB specimens of 355 patients with either SS or stomatitis sicca were compared with the smoking habits of the patients, and a lower focus score was found in patients who smoked. It appears, however, that external factors may influence the lymphocytic infiltrate seen in SLGB specimens. Medication may well be another external factor which influences the lymphocytic infiltrate. These factors should be taken into account when interpreting the SLGB results. 
This case report describes a patient with SS in whom changes occurred in the lymphocytic infiltrate in the SLGB specimen after treatment with corticosteroids.

\section{Case report}

A 35 year old man with Klinefelter's syndrome was referred with signs suggestive of SS. He complained of dry eyes and mouth, both of which had been present for 10 months. Shortly after onset of the dry eyes he had started to use artificial tear droplets. He also suffered from fatigue and had a history of back pain and a painful but not swollen left hand for 10 years. For the previous 8 months he had also had a painful left knee. He did not smoke or use any medication. Physical examination revealed no abnormalities other than signs compatible with Klinefelter's syndrome (gynaecomastia and spare body hair pattern), except for increased conjunctival injection. Laboratory findings included erythrocyte sedimentation rate (ESR) of $10 \mathrm{~mm} / 1 \mathrm{st} \mathrm{h}, \mathrm{C}$ reactive protein (CRP) $<5 \mathrm{mg} / \mathrm{l}$, normal blood count, normal liver function tests, normal kidney function, and slightly raised gammaglobulin $(16.6 \mathrm{~g} / \mathrm{l})$ without paraproteins on immunoelectrophoresis. Further testing revealed positive antinuclear antibody staining on indirect immunofluorescence with a homogeneous pattern. Tests for rheumatoid factor were negative. No autoantibodies such as anti-SS-A, anti-SS-B, anti-Sm, anti-dsDNA, and anti-RNP could be detected either by immunoblotting or by counter immunoelectrophoresis. Ophthalmic examination revealed a keratoconjunctivitis sicca with abnormal rose bengal staining of the cornea, diminished tear drop break up time (3 seconds), and Schirmer's test of $0 \mathrm{~mm}$ in 5 minutes for both eyes. Histological examination of the four glands from the SLGB showed 1.0 lymphocyte aggregates per $4 \mathrm{~mm}^{2}$ - that is, a focus score of 1.0. Quantitative immunohistological examination gave \% IgA of 64 . Based on the European Study Group diagnostic criteria, a diagnosis of SS was established.

Local treatment with eye droplets was started to treat the keratoconjunctivitis sicca. After several weeks, however, the patient developed a painless dystonia of the left hand, with the third to fifth finger as well as the wrist in fixed hyperflexion. The left foot showed a slight inversion and internal rotation. Serological testing for anti-dsDNA, antineutrophilic cytoplasmic, and anticardiolipin antibodies and lupus anticoagulants appeared to be negative. Further neurological examination showed no abnormalities by electromyography (EMG) or magnetic resonance imaging (MRI) of the brain. However, single photon emission computed tomography (SPECT) with iodobenzamide (IBZM) labelled with iodine-123 revealed decreased uptake in the right striatum (striatum:occipital cortex ratio 1.53 on the left, 1.41 on the right, mean normal (2SD) value $1.66(0.16))$, indicating a loss of D2 receptors in the right striatum (neurological details have been published previously). ${ }^{12} \mathrm{~A}$ diagnosis of neurovasculitis located in the right striatum was made, and was considered to be an extraglandular complication of SS. Treatment with high dose prednisone (60 mg daily) was started and within 2 weeks the dystonia and the sicca symptoms had disappeared. Re-examination of the lachrymal glands showed a normal Schirmer's test ( $15 \mathrm{~mm}$ in 5 minutes for both eyes), normal tear drop break up time, and normal rose bengal staining of the cornea. After 2 months of treatment the medication was tapered to a dose of $30 \mathrm{mg} /$ day. At that time, and after informed consent had been obtained, a second SLGB specimen was taken to examine the histological and immunohistological changes in the lymphocytic infiltrate. This specimen, in contrast to that taken before treatment, showed not only a normal focus score (0.4) but also a normal IgA \% score of 80 on examination of the four glands. Thus, in addition to an improvement in the clinical symptoms, the lymphocytic infiltrate appeared to have normalised after corticosteroid treatment. After 1 month, however, there was a relapse in both sicca and hand symptoms on prednisone $30 \mathrm{mg}$ daily. The dose of prednisone was increased to $60 \mathrm{mg}$ daily and azathioprine was added to enable the corticosteroid dosage to be re-tapered. Again the symptoms disappeared and returned after tapering of the prednisone dose. Azathioprine was therefore stopped and replaced with cyclophosphamide (100 mg daily) which enabled the corticosteroid dose to be successfully tapered.

\section{Discussion}

This case report describes a patient with SS complicated by neurovasculitis, manifested by dystonia of the left hand and foot. After treatment with high doses of corticosteroids, both the sicca symptoms and the dystonia quickly disappeared. Furthermore, at the same time this treatment resulted in normalisation of the histological and immunohistological abnormalities in the SLGB specimen. This suggests a relation between changes in clinical symptoms and histological and immunohistological changes. Repeated SLGBs might therefore offer an objective, target organ specific method for evaluating treatment since both the clinical and (immuno)histological signs appear to be reversible. This is important because there is, as yet, no clear marker of disease activity for SS and evaluation of treatment is primarily based on subjective parameters.

The histological findings of an SLGB specimen already play an important part in several criteria sets for SS. The diagnosis of SS was made because the patient met the following four of the six ESG criteria: the presence of ocular and oral symptoms for more than 3 months, ocular signs (abnormal Schirmer's test, abnormal rose bengal staining), and a focus score of 1.0. In the second SLGB specimen, however, a normal focus score was observed. If this had been the initial SLGB specimen, the diagnosis of SS would have been less likely and could have been missed, particularly since, at that time, the sicca signs had disappeared. Although the normalised second SLGB specimen might be 
found coincidentally as a result of sampling error, examination of both specimens included four glands and both samples were examined by two independent pathologists. Furthermore, Bodeutsch et al showed that the \%IgA containing plasma cells are not necessarily associated with lymphocytic foci and, in contrast to the focally situated lymphocyte aggregates, are much more diffusely distributed throughout the tissue of the minor salivary glands. ${ }^{7}$ Thus, the sampling error with regard to the \% IgA containing plasma cells is much less than that for the focus score. In our opinion, therefore, normalisation of both the focus score and the $\operatorname{IgA} \%$ score gives a reliable result.

This case shows that the effect of corticosteroids on the extent and composition of the infiltrate in the target organ in patients with SS cannot be excluded. The same phenomenon might appear when other immunosuppressive agents such as disease modifying antirheumatic drugs (DMARDs) are used. To minimise the potential bias of immunosuppressive agents, it is therefore important to take the SLGB specimen before starting antiinflammatory treatment. As patients with rheumatoid arthritis often also suffer from SS, the possible impact of treatment with DMARDs or corticosteroids on histological and quantitative immunohistological examination of an SLGB specimen should be taken into consideration in the diagnosis of SS. Since SLGB plays an important part in the diagnosis of SS, further studies need to be performed to establish which factors contribute to the composition of the infiltrate in these exocrine glands. However, the suggested dynamic properties of histopathological changes need to be confirmed in further studies.

1 Daniels TF, Aufdemorte ThB, Greenspan JS. Histopathology of Sjögren's syndrome. In: Talal N, Moutsopoulos HM, ogy of Sjögren's syndrome. In: Talal N, Moutsopoulos HM, aspects. Berlin: Springer Verlag, 1987:41-52.

2 Kassan SS, Thomas TL, Moutsopoulos HM, Hoover R, Kimberly RP, Budman DR, et al. Increased risk of ymphoma in sicca syndrome. Ann Intern Med 1978;89: $888-92$.

3 Vitali C, Bombardieri S, Moutsopoulos HM, Coll J, Gerli R, Hatron PY, et al. Assessment of the European classification criteria for Sjogren's syndrome in a series of clinically defined cases: results of a prospective multicentre study. The European Study Group on Diagnostic Criteria for The European Study Group on Diagnostic Criteria

4 Daniels TE. Clinical assessment and diagnosis of immunologically mediated salivary gland disease in Sjogren's syndrome. J Autoimmun 1989;2:529-41.

5 Fox RI, Saito I. Criteria for diagnosis of Sjogren's syndrome. Rheum Dis Clin North Am 1994;20:391-407.

6 Homma M, Tojo T, Akizuki M,Yamagata H. Criteria for Sjogren's syndrome in Japan. Scand J Rheumatol (suppl) 1986;61:26-7.

7 Bodeutsch C, de Wilde PC, Kater L, van Houwelingen JC, van den Hoogen FH, Kruize AA, et al. Quantitative immunohistologic criteria are superior to the lymphocytic focus nohistologic criteria are superior to the lymphocytic focus
score criterion for the diagnosis of Sjogren's syndrome. score criterion for the diagnosis of
Arthritis Rheum 1992;35:1075-87.

8 Lindahl G, Hedfors E. Lymphocytic infiltrates and epithelial HLA-DR expression in lip salivary glands in connective tissue disease patients lacking sicca: a prospective study. $\mathrm{Br}$ J Rheumatol 1989;28:293-8

9 Bodeutsch C, de Wilde PC, Kater L, Hene RJ, van den Hoogen FH, van de Putte LB, et al. Labial salivary gland biopsy in Sjogren's syndrome. Neth J Med 1992;40:148-57.

10 Speight PM, Cruchley A, Williams DM. Quantification of plasma cells in labial salivary glands: increased expression plasma cells in labial salivary glands: increased expression of IgM in $126-30$.

11 Manthorpe R, Benoni C, Jacobsson L, Kirtava Z, Larsson $\mathrm{A}$, Liedholm R, et al. Lower frequency of focal lip sialadenitis (focus score) in smoking patients. Can tobacco diminish the salivary gland involvement as judged by histological examination and anti-SSA/Ro and anti-SSB/La antibodies in Sjogren's syndrome? Ann Rheum Dis 2000;59:54-60.

12 Van den Berg JS, Horstink MW, van den Hoogen FH, Oyen WJ. Dystonia: a central nervous system presentation of Sjogren's syndrome. Mov Disord 1999;14:374-5. 